\title{
Seleção de famílias e progênies de irmãos completos de cana-de-açúcar para atributos tecnológicos e de produção pelo método de REML/BLUP
}

\author{
Mauro Alexandre Xavier ( $\left.{ }^{1}\right)$; Dilermando Perecin ( $\left.{ }^{2}\right)$; Karen Rodrigues de Toledo Alvim (2*); \\ Marcos Guimarães de Andrade Landell ('); Flávio Cese Arantes ( ${ }^{2}$ ) \\ (') Instituto Agronômico (IAC), Centro de Cana, Rodovia Antonio Duarte Nogueira, km 321, 14001-970 Ribeirão Preto (SP), Brasil. \\ (2) Universidade Estadual Paulista (UNESP), Faculdade de Ciências Agrárias e Veterinárias (FCAV), Departamento de Ciências Exatas, \\ Via de Acesso Prof. Paulo Donato Castellane, s/n, 14884-900 Jaboticabal (SP), Brasil. \\ (*) Autora correspondente: karenagro@yahoo.com.br
}

Recebido: 16/jun./2014; Aceito: 7/jul./2014

\begin{abstract}
Resumo
O objetivo deste trabalho foi avaliar as famílias e destacar as progênies superiores de cana-de-açúcar oriundas de 38 cruzamentos biparentais para os seguintes atributos: tonelada de colmos por hectare (TCH), toneladas de biomassa por hectare (TBIOH), brix (\% caldo da cana), teor de fibra, pureza, pol e açúcar total recuperável (ATR). Os dados foram analisados via modelos mistos REML/BLUP, em que o REML (Máxima Verossimilhança Restrita) permitiu estimar parâmetros genéticos e BLUP (Melhor Predição Linear Não Viciada) permitiu predizer os valores aditivos e genotípicos. A melhor família para os atributos TCH e TBIOH foi a 41, cujos parentais são as cultivares IACSP022019 x CTC9. Na seleção individual para TCH, a planta de n. 3 do bloco 2, do cruzamento 78 foi a que apresentou o melhor resultado. Para TBIOH, a planta n. 33, do bloco 1, da família 41 foi superior. As famílias 40, 41, 43, 68, 69, 79, 91, 92 e 147 foram superiores para as variáveis brix, pol, pureza e ATR, enquanto que as famílias 85, 147, 148, 149, 161, 163, 177, 178, 179 e 183 foram superiores para fibra. A família 147, cujos parentais são IACSP042286 x IACSP963055, mostrou três progênies entre as dez melhores tanto para brix quanto para fibra, o que identifica essa combinação como potencial fonte de progênies para produção de bioenergia.
\end{abstract}

Palavras-chave: Saccharum spp., modelos mistos, melhoramento genético.

\section{Selecting families and full-sib progenies of sugarcane for technological attributes and production by the method of REML/BLUP}

\begin{abstract}
The objective of this study was to assess families and highlight the superior progenies of sugarcane originating from 38 biparental crosses for the following attributes: tons of cane per hectare $(\mathrm{TCH})$, tons of biomass per hectare (TBIOH), brix (\% cane juice), fiber content, purity, pol and total recoverable sugar (TRS). The data were analyzed by mixed model REML / BLUP in the REML (Restricted Maximum Likelihood) allowed us to estimate genetic parameters and BLUP (best linear unbiased prediction) to predict the additive and genotypic values. The best family for the attributes TCH and TBIOH was 41, whose parents are cultivars IACSP022019 x CTC9. In individual selection for TCH, the plant number 3 of Block 2, the crossing 78, showed the best results. To TBIOH the plant number 33, Block 1, family 41, showed the best results. Families 40, 41, 43, 68, 69, 79, 91, 92 and147, were higher for the variables brix, pol, purity, and ATR, where as 85 families, 147, 148, 149, 161, 163, 177, 178, 179, and 183 were higher for fiber. The family 147 whose parents are IACSP042286 x IACSP963055, showed three progenies ranked among the top ten for both brix, and for fiber, which identifies the combination as a potential source of progenies for bioenergy production. Key words: Saccharum spp., mixed models, crop breeding.
\end{abstract}

\section{INTRODUÇÃO}

No Brasil, a área cultivada com cana-de-açúcar destinada à atividade sucroalcooleira está estimada em 8,37 milhôes de hectares. A previsão do total de cana moída na safra 2011/2012 é de 571,47 milhóes de toneladas, com queda de $8,4 \%$ em relação à safra 2010/2011, que foi de 623,90 milhôes de toneladas (CONAB, 2011).
O objetivo principal dos programas de melhoramento de cana-de-açúcar é abastecer o mercado com cultivares de interesse econômico, o que depende da variaçáo genética disponível na população de seleção e da criteriosa avaliação dos genótipos em experimentos bem delineados (Ramalho et al., 2005). Assim, os estudos desenvolvidos 
pelo Instituto Agronômico de Campinas (IAC) têm fornecido importantes subsídios técnicos para a expansáo da cultura no Estado de São Paulo com o desenvolvimento de cultivares de alto potencial produtivo e o aprimoramento dos critérios de seleção.

Em cana-de-açúcar, os cruzamentos mais utilizados são os biparentais, que se caracterizam por possibilitar a determinação tanto da capacidade específica quanto da capacidade geral de combinaçáo (CEC e CGC). Por outro lado, apresentam como limite para sua realização a falta de sincronismo de florescimento dos parentais.

Para Matsuoka et al. (2005), a herdabilidade entre famílias é superior àquela entre plantas individuais. No entanto, percebe-se que selecionando-se famílias sem considerar a variância genética dentro de cada cruzamento corre-se o risco de descartar indivíduos superiores em populaçóes com médias inferiores, mas com ampla variabilidade e assimetria favorável na distribuição das progênies (Moraes et al., 2010).

Oliveira et al. (2008) afirmam que a seleção de famílias por meio de modelos mistos REMLP/BLUP pode ser uma estratégia importante para identificar famílias com elevados valores genotípicos, em que haveria maior probabilidade de seleção de clones potenciais.

Além disso, é importante destacar que não só os atributos de produção, mas a análise tecnológica que avalia brix do caldo, teor de fibra, pureza, pol e açúcar total recuperável (ATR) torna-se imprescindível nas avaliaçóes para seleção tanto de famílias quanto de progênies, permitindo caracterização e possibilitando a identificação de genótipos com perfil varietal para a produção de bioenergia.

Sendo assim, o objetivo deste foi avaliar 38 famílias oriundas de cruzamentos biparentais em cana-de-açúcar pelo método dos modelos mistos - REML/BLUP - e destacar as famílias e progênies superiores para os atributos tonelada de colmos por hectare $(\mathrm{TCH})$, toneladas de biomassa por hectare (TBIOH), brix (\% caldo da cana), teor de fibra, pureza, pol e açúcar total recuperável (ATR).

\section{MATERIAL E MÉTOdOS}

O ensaio foi desenvolvido no Centro de Cana do IAC, localizado no Município de Ribeirão Preto, Estado de São Paulo, a uma altitude média de 531 metros do nível do mar, com relevo caracterizado como suavemente ondulado. Sua localização geográfica é definida como: latitude $21^{\circ} 12^{\prime} 42^{\prime \prime S}$ e longitude $47^{\circ} 48^{\prime} 24^{\prime \prime} \mathrm{W}$. O clima é do tipo tropical com inverno seco, classificado de acordo como o Sistema Internacional de Classificação de Köppen, como Aw. A pluviometria média anual é de $1.422 \mathrm{~mm}$.

Foram utilizadas 38 famílias de irmáos completos, cruzamentos biparentais plantados em dois blocos casualizados. Cada parcela foi constituída inicialmente por 40 plantas espaçadas de 1,5 m entre linhas e 0,6 m entre plantas, totalizando nos dois blocos 80 progênies por família e um total inicial, nas 38 famílias, de 3.080 progênies.

As famílias originam-se dos cruzamentos da série de hibridação 2009 do Instituto Agronômico de Campinas, realizada na Estação Experimental da Empresa Baiana de Desenvolvimento Agropecuário - EBDA, localizada na Ilha de Itaparica, BA, entre maio e junho de 2009. Os seedlings foram produzidos no Centro de Cana-de-Açúcar em Ribeirão Preto entre outubro e novembro e transplantados para o campo em 3 de dezembro 2009.

Tabela 1. Estimativas dos componentes de variância e parâmetros genéticos para atributos de produção (TCH e TBIOH) e de análise tecnológica (brix, pol, pureza, ATR e fibra) de 38 famílias de irmãos completos de cana-de-açúcar

\begin{tabular}{cccccccc} 
Parâmetros & TCH & TBIOH & Brix & Pol & Pureza & ATR & Fibra \\
\hline$\hat{\sigma}_{a}^{2}$ & 174,4474 & 72,8102 & 1,2226 & 1,0947 & 3,0801 & 89,5149 & 1,1486 \\
$\hat{\sigma}_{\text {parc }}^{2}$ & 125,6474 & 14,9660 & 0,5259 & 0,1609 & 1,2969 & 14,4932 & 0,1234 \\
\hline$\hat{\sigma}_{\text {fam }}^{2}$ & 51,19450 & 81,6701 & 0,0116 & 0,0144 & 0,0446 & 1,1453 & 0,0081 \\
\hline$\hat{\sigma}_{e}^{2}$ & 3211,0904 & 6633,6380 & 1,0664 & 0,9292 & 6,3983 & 77,2559 & 0,8484 \\
$\hat{\sigma}_{f}^{2}$ & 3562,3797 & 6803,0840 & 2,8265 & 2,1992 & 10,8200 & 182,4093 & 2,1285 \\
$\hat{h}_{a}^{2}$ & $0,0490 \pm$ & $0,0107 \pm$ & $0,4325 \pm$ & $0,4978 \pm$ & $0,2847 \pm$ & $0,4907 \pm$ & $0,5396 \pm$ \\
$\hat{h}_{g}^{2}$ & 0,0173 & 0,0081 & 0,1033 & 0,1108 & 0,0838 & 0,1100 & 0,1153 \\
$\hat{c}_{\text {parc }}^{2}$ & 0,1064 & 0,0587 & 0,4490 & 0,5239 & 0,3012 & 0,5158 & 0,5549 \\
$\hat{c}_{\text {fam }}^{2}$ & 0,0353 & 0,0022 & 0,1861 & 0,0731 & 0,1199 & 0,0795 & 0,0580 \\
\hline
\end{tabular}

$\hat{\sigma}_{a}^{2}$ : variância genética aditiva; $\hat{\sigma}_{\text {parc }}^{2}$ : variância ambiental entre parcelas; $\hat{\sigma}_{\text {fam }}^{2}$ : variância da capacidade específica de combinação ou variância genética de dominância entre famílias de irmấos completos; $\hat{\sigma}_{e}^{2}$ : variância residual; $\hat{\sigma}_{f}^{2}$ : variância fenotípica individual; $\hat{h}_{a}^{2}$ : herdabilidade individual no sentido restrito no bloco, ou seja, dos efeitos aditivos; $\hat{h}_{g}^{2}$ : herdabilidade individual no sentido amplo, ou seja, ajustada para os efeitos genotípicos totais; $\hat{c}_{\text {parc }}^{2}$ : coeficiente de determinaçáo dos efeitos de parcela; $\hat{c}_{\text {fam }}^{2}:$ coeficiente de determinação dos efeitos da capacidade específica de combinação. 
Os atributos agronômicos avaliados foram toneladas de colmo por hectare $(\mathrm{TCH})$, estimadas pela fórmula $T C H=\frac{d^{2} \times h \times n c(0,007854)}{0,4 x E}$, em que d $=$ diâmetro dos colmos, $\mathrm{h}=$ altura dos colmos; $\mathrm{nc}=$ número de colmos por touceira e $\mathrm{E}=1,5 \mathrm{~m}$ entrelinhas; toneladas de biomassa por hectare $(\mathrm{TBIOH})$ estimada por $\mathrm{TBIOH}=$ biomassa $\times(6,667 / 0,4)$, em que a biomassa quantifica-se em $\mathrm{kg}$ por touceira, colmos sem desponte. Em ambos os casos foi admitido que a touceira ocupou $0,4 \mathrm{~m}$ da linha, daí o valor 0,4 presente nas estimativas de $\mathrm{TCH}$ e TBIOH.

As variáveis da análise tecnológica investigadas foram: brix (\% caldo da cana), teor de fibra, pureza, pol e açúcar total recuperável (ATR), obtidas segundo

Tabela 2. Estimativa dos valores genotípicos do cruzamento (Vgc) das 38 famílias de irmãos completos de cana-de-açúcar para: toneladas de colmo por hectare $(\mathrm{TCH})$, tonelada de biomassa por hectare (TBIOH)

\begin{tabular}{|c|c|c|c|c|}
\hline \multirow{2}{*}{ Ordem } & \multicolumn{2}{|c|}{$\mathrm{TCH}$} & \multicolumn{2}{|c|}{ ТВІОН } \\
\hline & Família & Vgc & Família & Vgc \\
\hline 1 & 41 & 89,6490 & 41 & 124,9631 \\
\hline 2 & 78 & 84,8865 & 78 & 117,4225 \\
\hline 3 & 95 & 84,3465 & 1.029 & 115,9381 \\
\hline 4 & 79 & 82,9086 & 95 & 115,1444 \\
\hline 5 & 109 & 82,9062 & 109 & 113,8625 \\
\hline 6 & 161 & 82,4994 & 161 & 111,8869 \\
\hline 7 & 92 & 80,0658 & 149 & 111,4706 \\
\hline 8 & 1.029 & 77,8852 & 92 & 111,1550 \\
\hline 9 & 69 & 77,1202 & 40 & 110,9988 \\
\hline 10 & 113 & 76,8566 & 79 & 110,5569 \\
\hline 11 & 151 & 76,7023 & 91 & 109,3738 \\
\hline 12 & 43 & 76,1190 & 43 & 109,3013 \\
\hline 13 & 40 & 75,3931 & 80 & 109,2663 \\
\hline 14 & 149 & 74,1644 & 113 & 109,0494 \\
\hline 15 & 80 & 73,6631 & 177 & 108,4138 \\
\hline 16 & 163 & 72,9083 & 69 & 108,1844 \\
\hline 17 & 148 & 71,8547 & 42 & 107,6825 \\
\hline 18 & 152 & 71,8117 & 516 & 106,8238 \\
\hline 19 & 546 & 70,4011 & 152 & 106,2081 \\
\hline 20 & 91 & 70,3598 & 151 & 103,9844 \\
\hline 21 & 94 & 69,4194 & 90 & 103,9531 \\
\hline 22 & 42 & 69,4146 & 558 & 103,8225 \\
\hline 23 & 183 & 69,1110 & 183 & 103,5806 \\
\hline 24 & 147 & 68,9980 & 94 & 103,3675 \\
\hline 25 & 84 & 67,8772 & 179 & 101,9606 \\
\hline 26 & 85 & 67,2907 & 180 & 101,8138 \\
\hline 27 & 68 & 66,1829 & 546 & 101,7681 \\
\hline 28 & 516 & 66,0426 & 147 & 101,7381 \\
\hline 29 & 90 & 65,5789 & 148 & 100,8463 \\
\hline 30 & 185 & 65,3160 & 110 & 100,1088 \\
\hline 31 & 110 & 63,6921 & 163 & 97,69063 \\
\hline 32 & 558 & 62,9824 & 84 & 97,28313 \\
\hline 33 & 179 & 62,0670 & 185 & 96,98063 \\
\hline 34 & 178 & 60,2752 & 68 & 96,62938 \\
\hline 35 & 177 & 59,5509 & 178 & 94,44188 \\
\hline 36 & 180 & 58,3829 & 85 & 93,37313 \\
\hline 37 & 184 & 56,0887 & 184 & 89,00188 \\
\hline 38 & 182 & 53,8367 & 182 & 87,7125 \\
\hline$\mu$ & & 71,1739 & & 105,2044 \\
\hline
\end{tabular}

Em que, 40: IACCTC062785 x CTC9; 41: IACSP022019 x CTC9; 42: IACCTC063732 x CTC9; 43: IACCTC053543 x CTC9; 68: IACSP996308 x CTC9; 69: IACSP993013 CTC9; 78: IACSP032030 x IACSP952326; 79: IACSP032107 x IACSP952326; 80: IACSP03-2032 x IACSP95-2326; 84: IACCTC052511 x IACSP963055; 85 : IACCTC053546 x IACSP963055; 90: IACCTC063726 x CTC9; 91: IACCTC063747 x CTC9; 92: IACCTC063728 x CTC9; 94: CT973060 x IACSP944004; 95: SP842025 x IACSP944004; 109: IACSP022006 x CTC4; 110: IACSP022141 x CTC4; 113: IACSP032014 x CTC1; 147: IACSP042286 x IACSP963055; 148: IACSP042081 x IACSP963055; 149: CTC8 x IACSP963055; 151: IACCTC063738 x IACSP987019; 152: IACSP042081 x IACSP987019; 161: IACSP982072 x IACSP985010; 163: IACSP961107 x IACSP985010; 177: IACSP032082 x IACSP993009; 178: IACSP023157 x IACSP993009; 179: IACSP022095 x IACSP993009; 180: IACSP963130 x SP832847; 182: IACSP042150 x SP832847; 183: IACSP042065 x IACSP973391; 184: CT973060 x IACSP973391; 185: IACCTC063547 x IACSP973391; 516: IACSP955000 x CT024940; 546: CT961414 x SP88721; 558: IACSP963060 x IACSP933046; 1.029: IACSP963060 x CT961449; (família: fêmea x macho); $\mu$ : média geral do experimento. 
as normas técnicas de determinação da qualidade da cana-de-açúcar, conforme estabelece o Conselho de Produtores de Cana-de-Açúcar e Álcool do Estado de Sáo Paulo (CONSECANA, 2006).

Os dados foram analisados via modelos mistos REML/ BLUP, em que REML (Máxima Verossimilhança Restrita) estima parâmetros genéticos e BLUP (Melhor Predição Linear Não Viciada) prediz os valores aditivos e genotípicos, ordenando tanto as famílias como os indivíduos em relação aos atributos avaliados. Utilizou-se o delineamento de blocos completos, várias plantas por parcelas e genitores não aparentados, usando-se o software Selegen (modelo 33)
(Resende, 2002), que considera o seguinte modelo estatístico: $\mathrm{y}=\mathrm{Xr}+\mathrm{Za}+\mathrm{Wp}+\mathrm{Td}+\mathrm{e}$, em que y é o vetor de dados, $r$ é o vetor dos efeitos de repetiçáo (assumidos como fixos) somados à média geral, a é o vetor dos efeitos genéticos aditivos individuais (assumidos como aleatórios), p é o vetor dos efeitos de parcela (aleatórios), d é o vetor dos efeitos de dominância de família de irmãos germanos (aleatórios) e é o vetor de erros ou resíduos (aleatórios). As letras maiúsculas representam as matrizes de incidência para os referidos efeitos.

As famílias foram classificadas por seu valor genotípico, além disso foram classificadas as 25 melhores progênies entre

Tabela 3. Estimativa dos valores genotípicos do cruzamento ( $\mathrm{Vgc}$ ) das 38 famílias de irmãos completos de cana-de-açúcar para as variáveis brix (\% do caldo da cana), pol, pureza, teor de fibra, e açúcar total recuperável (ATR)

\begin{tabular}{|c|c|c|c|c|c|c|c|c|c|c|}
\hline \multirow{2}{*}{ Ordem } & \multicolumn{2}{|c|}{ BRIX } & \multicolumn{2}{|c|}{ POL } & \multicolumn{2}{|c|}{ PUREZA } & \multicolumn{2}{|c|}{ FIBRA } & \multicolumn{2}{|c|}{ ATR } \\
\hline & Família & Vgc & Família & Vgc & Família & Vgc & Família & Vgc & Família & Vgc \\
\hline 1 & 91 & 21,70 & 91 & 15,34 & 43 & 88,57 & 147 & 17,03 & 91 & 150,40 \\
\hline 2 & 79 & 21,37 & 79 & 15,26 & 42 & 87,87 & 85 & 16,96 & 79 & 149,65 \\
\hline 3 & 147 & 21,30 & 43 & 15,22 & 79 & 87,82 & 148 & 16,82 & 43 & 149,17 \\
\hline 4 & 41 & 21,28 & 41 & 14,88 & 41 & 87,73 & 149 & 16,40 & 41 & 146,18 \\
\hline 5 & 69 & 21,26 & 42 & 14,87 & 91 & 87,72 & 183 & 16,37 & 42 & 146,09 \\
\hline 6 & 90 & 21,23 & 69 & 14,80 & 92 & 87,71 & 161 & 16,32 & 69 & 145,63 \\
\hline 7 & 43 & 21,15 & 90 & 14,72 & 68 & 87,52 & 163 & 16,25 & 90 & 144,87 \\
\hline 8 & 68 & 21,14 & 40 & 14,71 & 40 & 87,49 & 177 & 16,20 & 40 & 144,70 \\
\hline 9 & 40 & 21,10 & 68 & 14,67 & 80 & 87,38 & 178 & 16,13 & 68 & 144,31 \\
\hline 10 & 84 & 21,10 & 84 & 14,65 & 69 & 87,35 & 179 & 16,07 & 84 & 144,07 \\
\hline 11 & 42 & 21,01 & 92 & 14,42 & 185 & 87,32 & 84 & 15,93 & 92 & 142,02 \\
\hline 12 & 148 & 20,91 & 147 & 14,36 & 90 & 87,28 & 68 & 15,93 & 147 & 141,55 \\
\hline 13 & 163 & 20,76 & 113 & 14,32 & 113 & 87,26 & 91 & 15,87 & 113 & 140,83 \\
\hline 14 & 85 & 20,73 & 185 & 14,30 & 84 & 86,93 & 90 & 15,86 & 185 & 140,67 \\
\hline 15 & 92 & 20,62 & 78 & 14,25 & 179 & 86,80 & 113 & 15,82 & 78 & 140,63 \\
\hline 16 & 78 & 20,52 & 80 & 14,23 & 152 & 86,79 & 41 & 15,81 & 80 & 140,21 \\
\hline 17 & 113 & 20,49 & 148 & 14,16 & 78 & 86,72 & 110 & 15,77 & 546 & 139,63 \\
\hline 18 & 161 & 20,44 & 546 & 14,11 & 151 & 86,63 & 184 & 15,75 & 148 & 139,61 \\
\hline 19 & 546 & 20,43 & 179 & 14,09 & 148 & 86,59 & 558 & 15,72 & 163 & 138,80 \\
\hline 20 & 80 & 20,41 & 163 & 14,05 & 85 & 86,48 & 69 & 15,67 & 179 & 138,75 \\
\hline 21 & 185 & 20,34 & 94 & 14,00 & 178 & 86,46 & 180 & 15,66 & 94 & 138,42 \\
\hline 22 & 183 & 20,30 & 95 & 13,97 & 147 & 86,14 & 152 & 15,63 & 95 & 138,11 \\
\hline 23 & 179 & 20,29 & 85 & 13,95 & 95 & 86,11 & 40 & 15,62 & 85 & 137,61 \\
\hline 24 & 109 & 20,26 & 152 & 13,83 & 110 & 86,00 & 185 & 15,38 & 109 & 137,01 \\
\hline 25 & 149 & 20,26 & 109 & 13,82 & 149 & 85,89 & 79 & 15,37 & 152 & 136,53 \\
\hline 26 & 94 & 20,25 & 151 & 13,78 & 183 & 85,86 & 80 & 15,35 & 151 & 136,13 \\
\hline 27 & 184 & 20,18 & 110 & 13,74 & 94 & 85,85 & 182 & 15,29 & 110 & 135,88 \\
\hline 28 & 95 & 20,15 & 184 & 13,66 & 184 & 85,84 & 78 & 15,08 & 184 & 135,28 \\
\hline 29 & 110 & 20,14 & 183 & 13,65 & 516 & 85,82 & 92 & 15,06 & 183 & 135,05 \\
\hline 30 & 152 & 20,09 & 161 & 13,60 & 163 & 85,61 & 42 & 15,04 & 161 & 134,84 \\
\hline 31 & 178 & 19,81 & 149 & 13,50 & 546 & 85,41 & 94 & 15,03 & 149 & 133,71 \\
\hline 32 & 151 & 19,79 & 178 & 13,48 & 1.029 & 85,41 & 109 & 14,94 & 178 & 133,26 \\
\hline 33 & 180 & 19,53 & 516 & 13,23 & 558 & 85,37 & 95 & 14,85 & 516 & 131,27 \\
\hline 34 & 558 & 19,46 & 1.029 & 13,21 & 109 & 85,09 & 43 & 14,83 & 1.029 & 131,11 \\
\hline 35 & 1.029 & 19,30 & 558 & 13,08 & 161 & 85,06 & 151 & 14,75 & 558 & 129,90 \\
\hline 36 & 177 & 19,23 & 180 & 13,00 & 177 & 84,95 & 546 & 14,59 & 180 & 129,35 \\
\hline 37 & 516 & 19,18 & 177 & 12,52 & 180 & 84,66 & 1.029 & 14,57 & 177 & 124,94 \\
\hline 38 & 182 & 19,06 & 182 & 12,47 & 182 & 84,05 & 516 & 14,33 & 182 & 124,78 \\
\hline$\mu$ & & 20,23 & & 13,87 & & 86,18 & & 15,51 & & 137,10 \\
\hline
\end{tabular}


as 38 famílias para cada atributo. Esse ranking individual foi feito pela média genotípica predita, que é o valor mais interessante do ponto de vista de ganho, por se trabalhar com uma cultura de propagação vegetativa.

\section{RESULTADOS E DISCUSSÃO}

As estimativas dos componentes de variância e parâmetros genéticos para os atributos avaliados encontram-se na tabela 1 . Observa-se que a variância genética aditiva é maior para os atributos de produção (TCH e TBIOH) e ATR, enquanto que a herdabilidade apresenta maiores valores para os atributos tecnológicos, corroborando Bressiani et al. (2002).

Considerando os valores genotípicos dos cruzamentos $(\mathrm{Vcg})$, a melhor família para os atributos TCH e TBIOH (Tabela 2) foi a 41, cujos parentais são as cultivares IACSP022019 x CTC9, com valores de 89,65 para TCH e de 124,96 para TBIOH.

Entre as dez melhores famílias, 41, 78, 79, 92, 95, 109, 161 e 1029 foram as melhores, tanto para TCH quanto para TBIOH (Tabela 2), sendo que os parentais masculinos CTC9 e IACSP952326 estão presentes em dois desses melhores cruzamentos. A indicação das melhores famílias pode permitir sua exploração de forma mais eficiente (Barbosa et al., 2004; 2005; Resende e Barbosa, 2006).

Já para os dados de análise tecnológica (Tabela 3), as famílias 40, 41, 43, 68, 69, 79 e 91 foram superiores para as variáveis brix, pol, pureza e ATR ao mesmo tempo. Para esse grupo de cruzamentos destaca-se novamente o parental CTC9, que aparece em $85 \%$ das melhores combinações. As famílias 85, 147, 148, 149, 161, 163, 177, 178, 179 e 183 foram superiores para o atributo fibra, com destaque para as frequências em que aparecem os parentais masculinos IACSP963055 e IACSP993009.

$\mathrm{Na}$ seleçáo individual para $\mathrm{TCH}$, todas as 25 plantas selecionadas pertencem a famílias que foram classificadas entre as dez melhores para o valor genotípico desse atributo. A planta de n. ${ }^{\circ} 3$ do bloco 2 do cruzamento 78 foi a melhor progênie, com valor genético aditivo predito e média genotípica predita de 88,73 e de 108,24, respectivamente (Tabela 4). Vale lembrar que a produtividade média brasileira está estimada em 68,29 TCH, segundo a CONAB (2011), dessa forma, a progênie citada tem potencial para produzir mais de $20 \%$ acima da média nacional.

De acordo com a tabela 5 , a planta $n .{ }^{\circ} 33$ do bloco 1 da família 41 foi superior para $\mathrm{TBIOH}$, com valor genético aditivo predito de 112,07 e média genotípica predita de

Tabela 4. Seleçáo das 25 melhores progênies oriundas das 38 famílias de irmãos completos de cana-de-açúcar para o atributo toneladas de colmo por hectare $(\mathrm{TCH})$

\begin{tabular}{|c|c|c|c|c|c|c|}
\hline \multicolumn{7}{|c|}{$\mathrm{TCH}$} \\
\hline Ordem & Bloco & Família & Planta & $f$ & $\mu+a$ & $\mu+g$ \\
\hline 1 & 2 & 78 & 3 & 418,0917 & 88,7292 & 108,2458 \\
\hline 2 & 2 & 78 & 1 & 369,4522 & 87,4429 & 104,6947 \\
\hline 3 & 2 & 1.029 & 32 & 443,4892 & 83,2449 & 103,6049 \\
\hline 4 & 2 & 95 & 4 & 344,1654 & 85,8359 & 102,7241 \\
\hline 5 & 1 & 43 & 1 & 441,9283 & 84,3787 & 102,6634 \\
\hline 6 & 1 & 41 & 33 & 267,6707 & 85,6019 & 102,3922 \\
\hline 7 & 2 & 95 & 7 & 335,9423 & 85,6185 & 102,1237 \\
\hline 8 & 1 & 41 & 35 & 247,2302 & 85,0614 & 100,8998 \\
\hline 9 & 2 & 161 & 2 & 309,5523 & 84,0879 & 99,4941 \\
\hline 10 & 1 & 161 & 30 & 323,4631 & 84,0181 & 99,3015 \\
\hline 11 & 2 & 78 & 13 & 295,3205 & 85,4825 & 99,2824 \\
\hline 12 & 1 & 41 & 17 & 217,8938 & 84,2856 & 98,7580 \\
\hline 13 & 2 & 41 & 1 & 223,3602 & 84,2134 & 98,5588 \\
\hline 14 & 2 & 41 & 40 & 221,6949 & 84,1694 & 98,4372 \\
\hline 15 & 1 & 161 & 5 & 309,0878 & 83,6379 & 98,2519 \\
\hline 16 & 2 & 41 & 27 & 216,4714 & 84,0312 & 98,0559 \\
\hline 17 & 2 & 41 & 38 & 212,7125 & 83,9318 & 97,7814 \\
\hline 18 & 1 & 41 & 9 & 202,2340 & 83,8715 & 97,6147 \\
\hline 19 & 2 & 41 & 2 & 205,2512 & 83,7345 & 97,2367 \\
\hline 20 & 2 & 41 & 21 & 202,6835 & 83,6666 & 97,0492 \\
\hline 21 & 1 & 78 & 1 & 242,3993 & 84,6457 & 96,9721 \\
\hline 22 & 1 & 109 & 21 & 279,8822 & 82,0417 & 96,8820 \\
\hline 23 & 2 & 79 & 31 & 267,3331 & 84,4037 & 96,8373 \\
\hline 24 & 2 & 41 & 6 & 196,3500 & 83,4991 & 96,5868 \\
\hline 25 & 2 & 41 & 7 & 196,1139 & 83,4929 & 96,5696 \\
\hline
\end{tabular}

f: valor fenotípico individual; $\mu+\mathrm{a}$ : valor genético aditivo predito; $\mu+\mathrm{g}$ : média genotípica predita. 
135,35. A família 41 foi a que apresentou maior frequência de progênies superiores, num total de $71 \%$.

Para a variável brix, as dez melhores progênies pertencem às famílias $40,43,69,79$ e 91 , sendo que $50 \%$ são da família 91, que possui os seguintes genitores: IACCTC063747 x CTC9. O melhor indivíduo para esse atributo foi o 21 da família 91 bloco 1, que apresentou valor fenotípico de 24,98, valor genético aditivo predito de 22,59 e média genotípica predita de 22,66 (Tabela 6). Destaca-se que plantas selecionadas de diversas famílias (Tabela 6) apresentaram média genotípica predita maior que o valor genotípico do melhor cruzamento, que foi de 21,70, da família 91; ou seja, isso mostra que é possível selecionar progênies superiores oriundas de cruzamentos com médias inferiores.

Para o atributo pol, os melhores indivíduos pertencem às famílias 42, 43, 79 e 91, sendo que as famílias 43, 79 e 91 correspondem a cerca de $80 \%$ desse total. O melhor indivíduo foi o 6 da família 79 do bloco 1 . Esse apresentou valor fenotípico de 17,72 , valor genético aditivo predito de 16,18 e média genotípica predita de 16,30 (Tabela 7).

Com relação à seleção individual para pureza, as famílias que apresentaram os melhores indivíduos foram 41, 43 e 91. Sendo que a família 43 apresentou $80 \%$ dos dez melhores indivíduos. $\mathrm{O}$ indivíduo superior foi o n. ${ }^{\circ} 10$ do bloco 1 da família 43, com 96,17 de valor fenotípico, 89,92 de valor genético aditivo predito e 90,09 de média genotípica predita (Tabela 8).

O indivíduo superior para ATR foi a planta 6 do bloco 1 da família 79, com $172,56 \mathrm{~kg}_{\text {ton }^{-1}}$ de valor fenotípico, $158,10 \mathrm{~kg} \mathrm{ton}^{-1}$ de valor genético aditivo predito e $159,16 \mathrm{~kg} \mathrm{ton}^{-1}$ de média genotípica predita. Os dez indivíduos superiores são das famílias 42, 43, 79 e 91 (Tabela 9), dessas, o parental masculino CTC 9 está presente em dois cruzamentos (43 e 91), reforçando a qualidade desse parental. As 25 progênies superiores (Tabela 9) mostram ganhos preditos superiores a $15 \mathrm{~kg}$ ton $^{-1}$ de ATR em relação ao previsto, 135,94, para a safra 2011/12 (CONAB, 2011).

Quanto à fibra, a melhor progênie é a n. ${ }^{\circ} 10$ da família 147 do bloco 1 , com valor fenotípico de $19,97 \%$, valor genético aditivo predito de $18,06 \%$ e média genotípica predita de $18,12 \%$. As famílias que mais apresentaram indivíduos superiores foram 85, 147, 148 e 163 (Tabela 10).

As progênies da família 147 originadas da combinação entre os parentais IACSP042286 x IACSP963055 associaram altos teores de sacarose e fibra, dessa forma, esse cruzamento é uma provável fonte para a seleçáo de cultivares modernas que atendam ao perfil bioenergético.

Tabela 5. Seleção das 25 melhores progênies oriundas das 38 famílias de irmãos completos de cana-de-açúcar para o atributo toneladas de biomassa por hectare $(\mathrm{TBIOH})$

\begin{tabular}{|c|c|c|c|c|c|c|}
\hline \multicolumn{7}{|c|}{ ТВІОН } \\
\hline Ordem & Bloco & Família & Planta & f & $\mu+a$ & $\mu+g$ \\
\hline 1 & 1 & 41 & 33 & 370,0185 & 112,0665 & 135,3507 \\
\hline 2 & 1 & 41 & 35 & 350,0175 & 111,9573 & 134,5068 \\
\hline 3 & 2 & 78 & 1 & 506,6920 & 111,5437 & 134,0663 \\
\hline 4 & 2 & 41 & 38 & 306,6820 & 111,7448 & 132,8638 \\
\hline 5 & 1 & 41 & 17 & 306,6820 & 111,7208 & 132,6785 \\
\hline 6 & 2 & 95 & 20 & 490,0245 & 110,0975 & 131,2189 \\
\hline 7 & 2 & 41 & 1 & 263,3465 & 111,5083 & 131,0354 \\
\hline 8 & 2 & 78 & 13 & 433,3550 & 111,1434 & 130,9721 \\
\hline 9 & 2 & 41 & 40 & 256,6795 & 111,4719 & 130,7541 \\
\hline 10 & 2 & 78 & 3 & 420,0210 & 111,0706 & 130,4096 \\
\hline 11 & 2 & 41 & 27 & 246,6790 & 111,4173 & 130,3322 \\
\hline 12 & 1 & 41 & 31 & 246,6790 & 111,3933 & 130,1469 \\
\hline 13 & 2 & 41 & 2 & 233,3450 & 111,3445 & 129,7696 \\
\hline 14 & 2 & 41 & 9 & 233,3450 & 111,3445 & 129,7696 \\
\hline 15 & 2 & 41 & 6 & 230,0115 & 111,3263 & 129,6290 \\
\hline 16 & 2 & 41 & 25 & 230,0115 & 111,3263 & 129,6290 \\
\hline 17 & 1 & 41 & 9 & 233,3450 & 111,3205 & 129,5843 \\
\hline 18 & 1 & 41 & 37 & 230,0115 & 111,3023 & 129,4437 \\
\hline 19 & 1 & 41 & 40 & 230,0115 & 111,3023 & 129,4437 \\
\hline 20 & 2 & 41 & 7 & 223,3445 & 111,2899 & 129,3477 \\
\hline 21 & 1 & 41 & 36 & 223,3445 & 111,2660 & 129,1624 \\
\hline 22 & 2 & 41 & 21 & 216,6775 & 111,2535 & 129,0664 \\
\hline 23 & 1 & 41 & 8 & 216,6775 & 111,2296 & 128,8811 \\
\hline 24 & 2 & 41 & 11 & 203,3435 & 111,1808 & 128,5038 \\
\hline 25 & 1 & 41 & 13 & 206,6770 & 111,1750 & 128,4592 \\
\hline
\end{tabular}

f: valor fenotípico individual; $\mu+\mathrm{a}$ : valor genético aditivo predito; $\mu+\mathrm{g}$ : média genotípica predita. 
Tabela 6. Seleçáo das 25 melhores progênies oriundas das 38 famílias de irmãos completos de cana-de-açúcar para o atributo brix (\% caldo da cana)

\begin{tabular}{|c|c|c|c|c|c|c|}
\hline \multicolumn{7}{|c|}{ BRIX } \\
\hline Ordem & Bloco & Família & Planta & $f$ & $\mu+a$ & $\mu+g$ \\
\hline 1 & 1 & 91 & 21 & 24,98 & 22,5928 & 22,6620 \\
\hline 2 & 1 & 79 & 6 & 24,78 & 22,5260 & 22,6146 \\
\hline 3 & 1 & 91 & 31 & 24,18 & 22,3013 & 22,3538 \\
\hline 4 & 1 & 91 & 35 & 23,66 & 22,1118 & 22,1535 \\
\hline 5 & 1 & 43 & 6 & 23,96 & 22,0627 & 22,1116 \\
\hline 6 & 1 & 91 & 36 & 23,49 & 22,0499 & 22,0880 \\
\hline 7 & 2 & 69 & 6 & 23,95 & 22,0318 & 22,0769 \\
\hline 8 & 1 & 91 & 18 & 23,27 & 21,9697 & 22,0033 \\
\hline 9 & 1 & 79 & 8 & 23,15 & 21,9321 & 21,9867 \\
\hline 10 & 2 & 40 & 14 & 23,30 & 21,9290 & 21,9711 \\
\hline 11 & 2 & 43 & 16 & 22,91 & 21,8483 & 21,8849 \\
\hline 12 & 1 & 147 & 7 & 22,50 & 21,8454 & 21,8907 \\
\hline 13 & 1 & 90 & 33 & 22,77 & 21,7899 & 21,8218 \\
\hline 14 & 2 & 79 & 31 & 23,66 & 21,7850 & 21,8313 \\
\hline 15 & 2 & 546 & 25 & 24,42 & 21,7740 & 21,8486 \\
\hline 16 & 1 & 41 & 36 & 22,38 & 21,7681 & 21,7978 \\
\hline 17 & 1 & 91 & 25 & 22,70 & 21,7620 & 21,7837 \\
\hline 18 & 2 & 147 & 22 & 23,80 & 21,7461 & 21,7857 \\
\hline 19 & 1 & 68 & 4 & 22,53 & 21,7388 & 21,7694 \\
\hline 20 & 1 & 69 & 28 & 22,35 & 21,7383 & 21,7666 \\
\hline 21 & 1 & 90 & 40 & 22,61 & 21,7316 & 21,7601 \\
\hline 22 & 2 & 79 & 20 & 23,51 & 21,7304 & 21,7735 \\
\hline 23 & 2 & 84 & 35 & 23,47 & 21,7238 & 21,7659 \\
\hline 24 & 2 & 147 & 24 & 23,61 & 21,6769 & 21,7125 \\
\hline 25 & 1 & 84 & 15 & 22,61 & 21,6660 & 21,7047 \\
\hline
\end{tabular}

f: valor fenotípico individual; $\mu+\mathrm{a}$ : valor genético aditivo predito; $\mu+\mathrm{g}$ : média genotípica predita.

Tabela 7. Seleção das 25 melhores progênies oriundas das 38 famílias de irmãos completos de cana-de-açúcar para o atributo pol

\begin{tabular}{|c|c|c|c|c|c|c|}
\hline \multicolumn{7}{|c|}{ POL } \\
\hline Ordem & Bloco & Família & Planta & $f$ & $\mu+a$ & $\mu+g$ \\
\hline 1 & 1 & 79 & 6 & 17,7193 & 16,1790 & 16,2962 \\
\hline 2 & 1 & 43 & 6 & 17,7574 & 16,1186 & 16,2134 \\
\hline 3 & 1 & 42 & 32 & 17,8276 & 16,0219 & 16,1178 \\
\hline 4 & 2 & 43 & 16 & 17,5975 & 15,9996 & 16,0850 \\
\hline 5 & 1 & 91 & 31 & 17,0586 & 15,9101 & 15,9856 \\
\hline 6 & 2 & 79 & 20 & 17,4668 & 15,8085 & 15,8965 \\
\hline 7 & 1 & 79 & 10 & 16,6635 & 15,7876 & 15,8740 \\
\hline 8 & 1 & 43 & 25 & 16,7789 & 15,7559 & 15,8221 \\
\hline 9 & 1 & 91 & 35 & 16,5418 & 15,7186 & 15,7790 \\
\hline 10 & 1 & 91 & 21 & 16,4823 & 15,6965 & 15,7552 \\
\hline 11 & 1 & 91 & 36 & 16,2074 & 15,5946 & 15,6452 \\
\hline 12 & 2 & 79 & 6 & 16,8591 & 15,5832 & 15,6535 \\
\hline 13 & 1 & 91 & 1 & 16,0708 & 15,5440 & 15,5906 \\
\hline 14 & 2 & 43 & 29 & 16,3616 & 15,5415 & 15,5908 \\
\hline 15 & 2 & 79 & 21 & 16,6473 & 15,5047 & 15,5688 \\
\hline 16 & 1 & 43 & 10 & 15,9838 & 15,4611 & 15,5041 \\
\hline 17 & 1 & 79 & 1 & 15,7444 & 15,4469 & 15,5064 \\
\hline 18 & 1 & 91 & 25 & 15,8020 & 15,4443 & 15,4831 \\
\hline 19 & 2 & 43 & 21 & 16,0329 & 15,4196 & 15,4593 \\
\hline 20 & 1 & 90 & 40 & 16,3404 & 15,4139 & 15,4657 \\
\hline 21 & 1 & 42 & 14 & 16,1806 & 15,4113 & 15,4591 \\
\hline 22 & 2 & 40 & 14 & 16,3875 & 15,3578 & 15,4055 \\
\hline 23 & 2 & 69 & 6 & 16,5297 & 15,3545 & 15,3995 \\
\hline 24 & 1 & 43 & 27 & 15,6905 & 15,3524 & 15,3868 \\
\hline 25 & 2 & 79 & 11 & 16,2316 & 15,3506 & 15,4026 \\
\hline
\end{tabular}

f: valor fenotípico individual; $\mu+\mathrm{a}$ : valor genético aditivo predito; $\mu+\mathrm{g}$ : média genotípica predita. 
Tabela 8. Seleção das 25 melhores progênies oriundas das 38 famílias de irmãos completos de cana-de-açúcar para o atributo pureza

\begin{tabular}{|c|c|c|c|c|c|c|}
\hline \multicolumn{7}{|c|}{ PUREZA } \\
\hline Ordem & Bloco & Família & Planta & f & $\mu+a$ & $\mu+g$ \\
\hline 1 & 1 & 43 & 10 & 96,17 & 89,9155 & 90,0920 \\
\hline 2 & 2 & 43 & 16 & 93,54 & 89,2279 & 89,3446 \\
\hline 3 & 2 & 43 & 11 & 91,29 & 88,7914 & 88,8701 \\
\hline 4 & 1 & 43 & 6 & 90,32 & 88,7806 & 88,8585 \\
\hline 5 & 1 & 43 & 27 & 90,27 & 88,7709 & 88,8479 \\
\hline 6 & 2 & 43 & 1 & 91,01 & 88,7371 & 88,8111 \\
\hline 7 & 1 & 43 & 25 & 90,04 & 88,7263 & 88,7994 \\
\hline 8 & 1 & 41 & 40 & 93,45 & 88,7170 & 88,8122 \\
\hline 9 & 1 & 91 & 1 & 93,02 & 88,7168 & 88,8122 \\
\hline 10 & 2 & 43 & 29 & 90,88 & 88,7119 & 88,7837 \\
\hline 11 & 1 & 42 & 14 & 92,47 & 88,6779 & 88,7658 \\
\hline 12 & 1 & 43 & 14 & 89,67 & 88,6545 & 88,7214 \\
\hline 13 & 1 & 79 & 10 & 92,67 & 88,6471 & 88,7645 \\
\hline 14 & 2 & 43 & 35 & 90,50 & 88,6381 & 88,7036 \\
\hline 15 & 2 & 79 & 14 & 93,12 & 88,6295 & 88,7454 \\
\hline 16 & 2 & 43 & 8 & 90,44 & 88,6265 & 88,6909 \\
\hline 17 & 2 & 43 & 36 & 90,35 & 88,6090 & 88,6719 \\
\hline 18 & 1 & 41 & 31 & 92,40 & 88,5133 & 88,5908 \\
\hline 19 & 2 & 92 & 30 & 91,82 & 88,4447 & 88,5168 \\
\hline 20 & 2 & 43 & 30 & 89,40 & 88,4247 & 88,4716 \\
\hline 21 & 1 & 91 & 31 & 91,48 & 88,4180 & 88,4875 \\
\hline 22 & 2 & 43 & 21 & 89,12 & 88,3704 & 88,4126 \\
\hline 23 & 2 & 80 & 1 & 93,09 & 88,3072 & 88,4071 \\
\hline 24 & 1 & 43 & 21 & 87,77 & 88,2859 & 88,3208 \\
\hline 25 & 2 & 43 & 20 & 88,67 & 88,2831 & 88,3177 \\
\hline
\end{tabular}

f: valor fenotípico individual; $\mu+a$ : valor genético aditivo predito; $\mu+\mathrm{g}$ : média genotípica predita.

Tabela 9. Seleção das 25 melhores progênies oriundas das 38 famílias de irmãos completos de cana-de-açúcar para o atributo ATR

\begin{tabular}{|c|c|c|c|c|c|c|}
\hline \multicolumn{7}{|c|}{ ATR } \\
\hline Ordem & Bloco & Família & Planta & $f$ & $\mu+a$ & $\mu+g$ \\
\hline 1 & 1 & 79 & 6 & 172,5633 & 158,1024 & 159,1609 \\
\hline 2 & 1 & 43 & 6 & 173,1950 & 157,5374 & 158,3981 \\
\hline 3 & 1 & 42 & 32 & 174,3651 & 156,9742 & 157,8665 \\
\hline 4 & 2 & 43 & 16 & 170,8536 & 156,2210 & 156,9807 \\
\hline 5 & 1 & 91 & 31 & 166,0176 & 155,4576 & 156,1287 \\
\hline 6 & 2 & 79 & 20 & 170,5181 & 154,7347 & 155,5347 \\
\hline 7 & 1 & 79 & 10 & 162,1461 & 154,2812 & 155,0463 \\
\hline 8 & 1 & 91 & 21 & 162,4942 & 154,1651 & 154,7370 \\
\hline 9 & 1 & 43 & 25 & 163,9866 & 154,1595 & 154,7609 \\
\hline 10 & 1 & 91 & 35 & 161,6031 & 153,8382 & 154,3850 \\
\hline 11 & 2 & 79 & 6 & 164,7651 & 152,6244 & 153,2623 \\
\hline 12 & 1 & 91 & 36 & 158,1813 & 152,5830 & 153,0334 \\
\hline 13 & 2 & 43 & 29 & 159,7787 & 152,1585 & 152,6062 \\
\hline 14 & 1 & 91 & 1 & 156,3958 & 151,9280 & 152,3282 \\
\hline 15 & 2 & 79 & 21 & 162,7891 & 151,8995 & 152,4818 \\
\hline 16 & 1 & 91 & 25 & 155,4379 & 151,5767 & 151,9498 \\
\hline 17 & 1 & 79 & 1 & 153,7824 & 151,2131 & 151,7427 \\
\hline 18 & 2 & 43 & 21 & 157,1189 & 151,1828 & 151,5557 \\
\hline 19 & 1 & 90 & 40 & 159,7279 & 151,1689 & 151,6451 \\
\hline 20 & 2 & 79 & 11 & 159,8707 & 150,8290 & 151,3291 \\
\hline 21 & 1 & 42 & 14 & 157,4726 & 150,7776 & 151,1942 \\
\hline 22 & 1 & 43 & 10 & 154,7652 & 150,7769 & 151,1186 \\
\hline 23 & 2 & 40 & 14 & 160,5178 & 150,7547 & 151,2033 \\
\hline 24 & 1 & 79 & 4 & 152,0960 & 150,5945 & 151,0766 \\
\hline 25 & 1 & 94 & 29 & 171,7118 & 150,5941 & 151,6904 \\
\hline
\end{tabular}

f: valor fenotípico individual; $\mu+\mathrm{a}$ : valor genético aditivo predito; $\mu+\mathrm{g}$ : média genotípica predita. 
Tabela 10. Seleção das 25 melhores progênies oriundas das 38 famílias de irmãos completos de cana-de-açúcar para o atributo fibra

\begin{tabular}{|c|c|c|c|c|c|c|}
\hline \multicolumn{7}{|c|}{ FIBRA } \\
\hline Ordem & Bloco & Família & Planta & f & $\mu+\mathbf{a}$ & $\mu+\mathrm{g}$ \\
\hline 1 & 1 & 147 & 10 & 19,97 & 18,064 & 18,1228 \\
\hline 2 & 2 & 85 & 11 & 19,71 & 18,0031 & 18,0603 \\
\hline 3 & 1 & 148 & 18 & 19,74 & 17,9954 & 18,0493 \\
\hline 4 & 2 & 85 & 13 & 19,58 & 17,9506 & 18,0056 \\
\hline 5 & 2 & 163 & 26 & 19,81 & 17,7765 & 17,8449 \\
\hline 6 & 1 & 85 & 35 & 18,94 & 17,699 & 17,7433 \\
\hline 7 & 2 & 148 & 1 & 18,94 & 17,6218 & 17,6599 \\
\hline 8 & 2 & 147 & 30 & 18,44 & 17,6104 & 17,6500 \\
\hline 9 & 1 & 147 & 7 & 18,79 & 17,5876 & 17,6263 \\
\hline 10 & 2 & 85 & 2 & 18,60 & 17,5550 & 17,5932 \\
\hline 11 & 1 & 148 & 22 & 18,62 & 17,5433 & 17,5780 \\
\hline 12 & 2 & 85 & 40 & 18,48 & 17,5066 & 17,5427 \\
\hline 13 & 2 & 148 & 9 & 18,62 & 17,4926 & 17,5252 \\
\hline 14 & 1 & 178 & 23 & 19,71 & 17,4768 & 17,5355 \\
\hline 15 & 1 & 85 & 30 & 18,09 & 17,3559 & 17,3857 \\
\hline 16 & 1 & 147 & 26 & 18,13 & 17,3212 & 17,3485 \\
\hline 17 & 2 & 147 & 34 & 17,72 & 17,3198 & 17,3470 \\
\hline 18 & 2 & 149 & 12 & 18,87 & 17,3022 & 17,3374 \\
\hline 19 & 2 & 148 & 30 & 18,10 & 17,2827 & 17,3064 \\
\hline 20 & 1 & 85 & 34 & 17,83 & 17,2509 & 17,2763 \\
\hline 21 & 2 & 85 & 31 & 17,84 & 17,2482 & 17,2734 \\
\hline 22 & 1 & 147 & 5 & 17,92 & 17,2364 & 17,2602 \\
\hline 23 & 2 & 147 & 39 & 17,45 & 17,2108 & 17,2334 \\
\hline 24 & 1 & 110 & 10 & 19,44 & 17,2103 & 17,2789 \\
\hline 25 & 2 & 148 & 34 & 17,90 & 17,2020 & 17,2223 \\
\hline
\end{tabular}

f: valor fenotípico individual; $\mu+\mathrm{a}$ : valor genético aditivo predito; $\mu+\mathrm{g}$ : média genotípica predita.

\section{CONCLUSÃO}

A melhor família para os atributos de produção foi a 41, cujos parentais são as cultivares IACSP022019 x CTC9.

$\mathrm{Na}$ seleção individual para TCH, a planta de n. 3 do bloco 2 do cruzamento 78 foi o melhor indivíduo. Enquanto que, para TBIOH, a planta n. ${ }^{\circ} 33$ do bloco 1 da família 41 foi o melhor indivíduo.

As famílias 40, 41, 43, 68, 69, 79, 91, 92 e 147 foram superiores para os atributos brix, pol, pureza e ATR.

As famílias 85, 147, 148, 149, 161, 163, 177, 178, 179 e 183 foram superiores para o atributo fibra.

A família 147 (IACSP042286 x IACSP963055) é uma provável fonte para a seleçáo de genótipos que atendam ao perfil bioenergético, por apresentar altos valores genotípicos $(\mathrm{Vcg})$ tanto para teor de sacarose quanto para fibra.

As cultivares CTC9 e IACSP962326 são boas parentais masculinas para os atributos tecnológicos.

\section{REFERÊNCIAS}

BARBOSA, M.H.P.; RESENDE, M.D.V.; PETERNELLI, L.A.; BRESSIANI, J.A.; SILVEIRA, L.C.I.; SILVA, F.L.; FIGUEIREDO, I.C.R. Use of REML/BLUP for the selection of sugarcane families specialized in biomass production. Crop Breeding and Applied Biotechnology, v.4, p.218-226, 2004.

BARBOSA, M.H.P.; RESENDE, M.D.V.; BRESSIANI, J.A.; SILVEIRA, L.C.I.; PETERNELLI, L.SA. Selection of sugarcane families and parents by REML/BLUP. Crop Breeding and Applied Biotechnology, v.5, p.443-450, 2005. http://dx.doi.org/10.12702/19847033.v05n04a 10

BRESSIANI, J.A.; VENCOVSKY, R.; BURNQUIST, W.L. Interação entre famílias de cana-de-açúcar e locais: efeito na resposta esperada com a seleçāo. Bragantia, v.61, p.1-10, 2002. http://dx.doi.org/10.1590/ S0006-87052002000100002

COMPANHIA NACIONAL DE ABASTECIMENTO - CONAB. Acompanhamento da safra brasileira. Brasília, 2011. Disponível em: $<$ http//www.conab.gov.br>.

CONSELHO DOS PRODUTORES DE CANA-DEAÇÚCAR, AÇÚCAR E ÁLCOOL DO ESTADO DE SÃO PAULO - CONSECANA. Manual de Instruçōes. Piracicaba, 2006. $112 \mathrm{p}$.

MATSUOKA, S.; GARCIA, A.A.F;; ARIZONO, H. Melhoramento da cana-de-açúcar. In: BORÉM, A. Melhoramento de espécies cultivadas. Viçosa: UFV, 2005. p.255-274.

MORAES, M.F.; BASTOS G.Q.; FILHO, C.J.A.; MELO, L.J.O.T.; REIS, O.V. Avaliaçáo agroindustrial e parâmetros genético de progênies de cana-de-açúcar em fase inicial na zona canavieira do litoral norte de Pernambuco. Ciência Agrotecnologia, v.34, p.1086-1092, 2010. http://dx.doi.org/10.1590/S1413-70542010000500002 
OLIVEIRA, R.A.; DAROS, E.; BESPALHOK-FILHO, J.C.; ZAMBON, J.L.C.; IDO, O.T.; WEBER, H.; RESENDE, M.D.V.; ZENI-NETO, H. Seleção de famílias de cana-de-açúcar via modelos mistos. Scientia Agraria, v.9, p.269-274, 2008.

RAMALHO, M.A.P.; FERREIRA, D.F.; OLIVEIRA, A.C. Experimentaçáo em genética e melhoramento de plantas. 2. ed. Lavras: UFLA, 2005. 326p.
RESENDE, M.D.V. Software SELEGEN - REML/BLUP. Colombo: EMBRAPA - CNPF, 2002. 67p. (Série Documentos, 77)

RESENDE, M.D.V.; BARBOSA, M.H.P. Selection via simulated individual BLUP basead on family genotypic effects in sugarcane. Pesquisa Agropecuária Brasileira, v.41, p.421-429, 2006. http:// dx.doi.org/10.1590/S0100-204X2006000300008 